Pacific Journal of Mathematic 


\title{
COMPOSITION SERIES IN CHEVALLEY ALGEBRAS
}

\section{JAMES F. HURLEY}

\begin{abstract}
This paper continues the study of how the ideal structure of a Chevalley algebra (a Lie algebra obtained by transferring the scalars of a finite dimensional simple Lie algebra over $C$ to a commutative ring $R$ with identity in which 2 and 3 are not zero divisors) depends on the ideal structure of $R$. Specifically, we find that composition series of ideals for the Chevalley algebras exist only in case $R$ has composition series of ideals, and in the latter case give explicit descriptions of the composition series in the Chevalley algebras. We also give a necessary and sufficient condition for the composition series in the algebra to exactly parallel those in the ring.
\end{abstract}

In two earlier papers ([3] and [4]), we have used the fundamental procedures of Chevalley [1] to construct certain Lie algebras from the finite dimensional complex simple algebras through replacement of the scalars by elements of a commutative ring $R$ with identity in which 2 and 3 are neither zero nor zero divisors. The main results of these papers concerned the question of to what extent simplicity of the original algebras reflects itself in the ideal structure of the new algebras, which we call Chevalley algebras. In the case when the ring $R$ is a field of prime characteristic, what amounts to this same question was previously considered by Dieudonné [2], Ono [7], and, as a tool for studying automorphisms, Steinberg [8]. The major emphasis in [2] however was upon the nature of the composition series of ideals in the nonsimple Chevalley algebras, with explicit results being obtained for the exceptional algebras and implicit results noted in the still earlier work of Jacobson ([5] and [6]). In the present paper, we take up this topic in the setting of an arbitrary commutative ground ring with identity, with our methods once more requiring exclusion of the cases when 2 or 3 are zero or zero divisors. We obtain results which give the extent to which the nature of composition series of ideals in the Chevalley algebras is determined by the nature of the composition series of ideals in the ring $R$.

Let $L$ be a simple Lie algebra of finite dimension over the complex field, $H$ an $n$-dimensional Cartan subalgebra, $\Sigma$ the (ordered) set of nonzero roots determined by $H$, and $I I$ the set of simple roots. For $r$ and $s$ in $\Sigma$, we denote the Cartan integer $2(r, s) /(s, s)$ by $c(r, s)$. When referring to the length of a root $r$, we shall mean simply $\sqrt{(r, r)}$.

Let $B=\left\{e_{r}\right\} \cup\left\{h_{i}\right\}$ be a Chevalley basis of $L$. Let $L_{z}$ be the free additive abelian group generated by $B$. Since the structural constants 
of $L$ relative to $B$ are all integers, if we define $L_{R}$ to be $R \otimes_{z} L_{Z}$, then $L_{R}$ can be viewed as a Lie algebra over $R$, where the multiplication table for $B$ is used with all integers interpreted in $R$. Under the obvious identification, we may regard $B$ as a basis of $L_{R}$.

Let $\left\{h_{1}^{\prime}, \cdots, h_{n}^{\prime}\right\}$ be a complex basis of $H$ which is dual to the system $\Pi=\left\{r_{1}, r_{2}, \cdots, r_{n}\right\}$. Then $H_{Z} \subseteq H_{Z}^{\prime}$ where $H_{Z}$ is the additive group generated by $\left\{h_{1}, \cdots, h_{n}\right\}$ and $H_{Z}^{\prime}$ is that generated by $\left\{h_{1}^{\prime}, \cdots, h_{n}^{\prime}\right\}$. In fact, $h_{i}=\sum_{j=1}^{n} c\left(r_{j}, r_{i}\right) h_{j}^{\prime}$ and $H_{R}=R \otimes H_{z} \subseteq H_{R}^{\prime}=R \otimes H_{z}^{\prime}$. There exist then basis $\left\{\bar{h}_{1}, \cdots, \bar{h}_{n}\right\}$ of $H_{R}$ and $\left\{\bar{h}_{1}^{\prime}, \cdots, \bar{h}_{n}^{\prime}\right\}$ of $H_{R}^{\prime}$ such that $\bar{h}_{i}=d_{i} \bar{h}_{i}^{\prime}$, with $d_{i}$ the $i^{\text {th }}$ elementary divisor of the Cartan matrix $C$ of $L$. In the sequel we also use $C$ to represent the linear transformation on $H$ whose matrix relative to $\left\{h_{1}, \cdots, h_{n}\right\}$ is the Cartan matrix of $L$. Recalling that a simple algebra has at most two distinct root lengths, we use $s$ and $t$ as generic symbols for short and long roots respectively, and define $E_{R}, E_{S}$, and $E_{L}$ to be the $R$-submodules of $L_{R}$ generated by $\left\{e_{r} \mid r \in \Sigma\right\},\left\{e_{s} \mid s\right.$ a short root $\}$ and $\left\{e_{t} \mid t\right.$ a long root $\} . H_{S}$ and $H_{L}$ are defined similarly.

The basic relationship between ideals in $L_{R}$ and ideals in $R$ tells us that only for a narrow class of our rings $R$ will $L_{R}$ possess a composition series. We remark first that the existence of a composition series of ideals in $L_{R}$ is equivalent to the presence of the ascending and descending chain conditions on ideals in $L_{R}$, since the lattice of ideals of $L_{R}$ is, as usual, modular. The following lemma, a consequence of this remark, now limits our ensuing discussion to rings having a composition series of ideals.

LEMMA. If $R$ is a ring with no composition series (in the sense of [9]), then $L_{R}$ has no composition series of ideals.

Proof. If $R$ has no composition series, then there exists an infinite sequence of ideals $J_{i}$ of $R$ which is either strictly increasing or strictly decreasing. It is then easily seen that the corresponding $J_{i} L_{R}$ are ideals in $L_{R}$ and together form an infinite sequence of the same sort as the $J_{i}$ form. Thus $L_{R}$ has no composition series of ideals.

In the sequel, the converse of this lemma is essentially obtained through consideration first of Lie algebras $L$ of one root length, then nonsymplectic $L$ with two root lengths, and finally symplectic $L$. We in fact obtain explicit characterizations of composition series in $L_{R}$ in terms of a given composition series in $R$.

2. Statement of results. Let

$$
L_{R}=M_{0} \supset M_{1} \supset \cdots \supset M_{m} \supset M_{m+1}=0
$$


be a composition series of ideals for $L_{R}$. We say that this composition series is determined by a composition series in $R$ if there exists a composision series $R=J_{0} \supset J_{1} \supset \cdots \supset J_{m} \supset J_{m+1}=0$ such that for each $i, M_{i}=J_{i} L_{R}$.

THEOREM 1. A necesssary and sufficient condition for every composition series in $L_{R}$ to be determined by one in $R$ is that $\operatorname{det} C$ and $(t, t) /(s, s)$ both be invertible in $R$.

Theorem 2. Let $L$ be of type $A_{n}, n \geqq 2, D_{n}, n$ even $\geqq 4, E_{6}$, or $E_{7}$. Then there exists a composition series $\left\{J_{0}, J_{1}, \cdots, J_{k}, J_{k+1}\right\}$ in $R$ such that

$$
\begin{aligned}
& M_{1}=J_{1} E_{R}+J_{1} \bar{h}_{1}+\cdots+J_{1} \bar{h}_{n-1}+R \bar{h}_{n}, \\
& M_{2}=J_{1} L_{R}, \\
& M_{3}=J_{2} E_{R}+J_{2} \bar{h}_{1}+\cdots+J_{2} \bar{h}_{n-1}+J_{1} \bar{h}_{n}, \\
& M_{4}=J_{2} L_{R}, \cdots M_{m-1}=J_{k} L_{R}, \quad M_{m}=J_{k} \bar{h}_{n} .
\end{aligned}
$$

If $L$ is of type $E_{8}$, then every composition series for $L_{R}$ is determined by one in $R$.

THeOREM 3. Let $L$ be of type $D_{n}, n \geqq 3$ odd. Then there exists a composition series $\left\{J_{0}, J_{1}, \cdots, J_{k}, J_{k+1}\right\}$ in $R$ such that

$$
\begin{aligned}
M_{1}= & J_{1} E_{R}+J_{1} \bar{h}_{1}+\cdots+J_{1} \bar{h}_{n-2}+R \bar{h}_{n-1}+R \bar{h}_{n}, \\
M_{2}= & J_{1} E_{R}+J_{1} \bar{h}_{1}+\cdots+J_{1} \bar{h}_{n-2}+J^{(1)} \bar{h}_{n-1}+J^{(2)} \bar{h}_{n} \text { where one of } \\
& J^{(1)}, J^{(2)} \text { is } R \text { and the other is } J_{1}, \\
M_{3}= & J_{1} L_{R}, \\
M_{4}= & J_{2} E_{R}+J_{2} \bar{h}_{1}+\cdots+J_{2} \bar{h}_{n-2}+J^{(3)} \bar{h}_{n-1}+J^{(4)} \bar{h}_{n} \text { where one of } \\
& J^{(3)}, J^{(4)} \text { is } J_{1} \text { and the other is } J_{2}, \\
\vdots & \\
M_{m-1}= & J_{k} \bar{h}_{n-1}+J_{k} \bar{h}_{n}, \\
M_{m}= & J_{k} \bar{h}_{n-1} \text { or } J_{k} \bar{h}_{n} .
\end{aligned}
$$

THEOREM 4. Let $L$ be of type $B_{n}, n \geqq 3$. Let $\left\{\bar{h}_{i}\right\}$ be the basis of Theorem 7.3 of [3]. Then there exists a composition series $\left\{J_{0}, J_{1}, \cdots, J_{k}, J_{k+1}\right\}$ in $R$ such that

$$
\begin{aligned}
M_{1}= & J_{1} E_{L}+R E_{S}+R \bar{h}_{1}+J_{1} \bar{h}_{2}+R \bar{h}_{3}+J_{1} \bar{h}_{4}+\cdots+J_{1} \bar{h}_{n-1}+R \bar{h}_{n}, \\
M_{2}= & J_{1} E_{L}+R E_{S}+R \bar{h}_{1}+J_{1} \bar{h}_{2}+J_{1} \bar{h}_{3}+J_{1} \bar{h}_{4}+\cdots+J_{1} \bar{h}_{n-1}+R \bar{h}_{n}, \\
& \text { or } J_{1} E_{L}+R E_{S}+R \bar{h}_{1}+J_{1} \bar{h}_{2}+R \bar{h}_{3}+J_{1} \bar{h}_{4}+\cdots+J_{1} \bar{h}_{n-1}+J_{1} \bar{h}_{n}, \\
M_{3}= & J_{1} E_{L}+R E_{S}+R \bar{h}_{1}+J_{1} \bar{h}_{2}+J_{1} \bar{h}_{3}+J_{1} \bar{h}_{4}+\cdots+J_{1} \bar{h}_{n-1}+J_{1} \bar{h}_{n}, \\
M_{4}= & J_{1} E_{R}+J_{1} \bar{h}_{1}+\cdots+J_{1} \bar{h}_{n-1}+R \bar{h}_{n},
\end{aligned}
$$




$$
\begin{aligned}
& M_{5}=J_{1} L_{R}, \\
& \vdots \\
& M_{m-2}=J_{k} L_{R}, \\
& M_{m-1}=J_{k} E_{S}+J_{k} \bar{h}_{1}+J_{k} \bar{h}_{3}+J_{k} \bar{h}_{n}, \\
& M_{m}=J_{k} \bar{h}_{n} .
\end{aligned}
$$

THEOREM 5. Let $L$ be of type $F_{4}$ and $\left\{\bar{h}_{i}\right\}$ be the basis of Theorem 7.5 of [3]. Then there exists a composition series $\left\{J_{0}, J_{1}, \cdots, J_{k}, J_{k+1}\right\}$ of ideals in $R$ such that

$$
\begin{aligned}
& M_{1}=J_{1} E_{L}+R E_{S}+R \bar{h}_{1}+R \bar{h}_{2}+J_{1} \bar{h}_{3}+J \bar{h}_{4}, \\
& M_{2}=J_{1} L_{R}, \\
& M_{3}=J_{2} E_{L}+J_{1} E_{S}+J_{1} \bar{h}_{1}+J_{1} \bar{h}_{2}+J_{2} \bar{h}_{3}+J_{2} \bar{h}_{4}, \\
& M_{4}=J_{2} L_{R}, \\
& \vdots \\
& M_{m-1}=J_{k} L_{R}, \\
& M_{m}=J_{k} E_{S}+J_{k} \bar{h}_{1}+J_{k} \bar{h}_{2} .
\end{aligned}
$$

THEOREM 6. Let $L$ be of type $G_{2}$ and let $\left\{\bar{h}_{i}\right\}$ be the basis of Theorem 7.7 of [3]. Then there is a composition series

$$
\left\{J_{0}, J_{1}, \cdots, J_{k}, J_{k+1}\right\}
$$

of $R$ such that

$$
\begin{aligned}
& M_{1}=J_{1} e_{1}+R e_{2}+J_{1} \bar{h}_{1}+R \bar{h}_{2}, \\
& M_{2}=J_{1} L_{R}, \\
& M_{3}=J_{2} e_{1}+J_{1} e_{2}+J_{2} \bar{h}_{1}+J_{1} \bar{h}_{2}, \\
& M_{4}=J_{2} L_{R}, \\
& \vdots \\
& M_{m-1}=J_{k} L_{R}, \\
& M_{m}=J_{k} e_{2}+J_{k} \bar{h}_{2} .
\end{aligned}
$$

3. Proof. The proofs of our results depend of course on the nature of the ideals in $L_{R}$, a characterization of which is found in [3]. When appropriate, we shall refer to results in [3] by number without giving the explicit statements themselves.

3.1. Proof of Theorem 1. By Theorem 3.3 of [3], every ideal in $L_{R}$ has the form $J L_{R}$ for some ideal $J$ in $R$ if and only if the two integers $\operatorname{det} C$ and $(t, t) /(s, s)$ are invertible in $R$. If these integers are invertible and a composition series $\left\{M_{0}, M_{1}, M_{2}, \cdots, M_{m}, M_{m+1}\right\}$ is 
given in $L_{R}$, then we have $M_{i}=J_{i} L_{R}$ for some ideal $J_{i}$ in $R, i=$ $1,2, \cdots, \mathrm{m}$. Since no ideals exist in $L_{R}$ between $M_{i}$ and $M_{i+1}$, neither do any exist in $R$ between $J_{i}$ and $J_{i+1}$. Hence $\left\{J_{0}, J_{1}, \cdots, J_{m}, J_{m+1}\right\}$ is a composition series in $R$ which determines the given series in $L_{R}$. Conversely, if every composition series in $L_{R}$ consists of terms of the form $J_{i} L_{R}$ where $\left\{J_{i}\right\}$ is some composition series in $R$, then no ideals can exist in $L_{R}$ which are not of the form $J L_{R}$ for some ideal $J$ in $R$. Then $\operatorname{det} C$ and $(t, t) /(s, s)$ are invertible in $R$.

3.2. Proof of Theorems 2 and 3. Given the composition series in $L_{R}$, we know that $M_{1}$, being a maximal ideal, has the asserted form for $J_{1}$ a maximal ideal in $R$, by virtue of Theorem 6.3 of [3] in all cases except $E_{8}$. For $E_{8}$ however, the conclusion of Theorem 1 is available since $\operatorname{det} C=1$ and there is only one root length. In view of Theorems 3.4 and 6.2 of [3], in order for no ideal of $L_{R}$ to exist between $M_{1}$ and $M_{2}$, it must be that $M_{2}$ has the asserted form also, and similarly for $M_{3}$ in the case $D_{n}, n$ odd. Again by the above quoted theorems, if no ideals in $L_{R}$ exist between $M_{2}$ and $M_{3}\left(M_{3}\right.$ and $M_{4}$ in case $D_{n}, n$ odd), then there must exist an ideal $J_{2}$ of $R$, with $J_{2}$ maximal among the ideals of $R$ contained in $J_{1}$ and having the property that $M_{3}\left(M_{4}\right.$ in case $D_{n}, n$ odd) has the asserted form. Repetition of this reasoning at each stage yields the desired composition series in $R$ and completes the proof.

3.3. Proof of Theorems 4,5, and 6. We reason as in 3.2, this time calling upon the relevant theorems in [3] for the nonsymplectic algebras of two root lengths. The maximal ideal $M_{1}$ has the form asserted for some maximal ideal $J_{1}$ in $R$ by appeal to Theorems 7.4, 7.6, and 7.8 of [3] in the respective cases $B_{n}, F_{4}$ and $G_{2}$. Since no ideals in $L_{R}$ exist between $M_{1}$ and $M_{2}$, we use Theorems 3.5, 7.3, 7.5, and 7.7 of [3] to determine the nature of $M_{2}$. We know in each case that $M_{2} \cap E_{R}=J_{1} E_{L}+R E_{S}$ and that

$$
J_{1} H_{L}+R H_{S} \leqq M_{2} \cap H_{R} \subseteq C^{-1}\left(R H_{S}+J_{1} H_{L}\right) \text {. }
$$

Thus to preclude ideals between $M_{1}$ and $M_{2}$ we need only make $M_{2} \cap H_{R}$ a maximal $R$-submodule of $M_{1} \cap H_{R}$, all in view of 3.5 of [3]. The subsequently listed results merely prescribe that $M_{2}$ then has the form asserted in Theorems 4,5 , and 6 in the respective cases $B_{n}, F_{4}$, and $G_{2}$. The same combination of references is effective in producing the ideals of $R$ needed to complete the composition series below $J_{1}$ and with it the proof.

4. The symplectic algebras. If $L$ is of type $C_{n}, n \geqq 2$, the ideal structure of $L_{R}$ is far less tidy than in the other cases, so much 
so that the concrete representations of the ideals (and so of the composition series of ideals) in $L_{R}$ given above in terms of simply chosen bases just no longer exist. Using Theorem 3.6 of [3] however, we can at least describe a composition series in $L_{R}$ module the nature of composition series of $R$-submodules in $H_{R}$. Since $M_{1}$ is a maximal ideal of $L_{R}$, we know that $M_{1} \cap E_{S}=J_{1} E_{S}$ for some maximal ideal $J$ of $R$. Moreover, $M_{1} \cap\left(E_{L}+H_{R}\right) \subseteq J_{1} E_{L}+C^{-1}\left(J_{1} H_{R}\right)$. By maximality then $M_{1}$ must be $J_{1} E_{R}+C^{-1}\left(J_{1} H_{R}\right)$. We have that $C^{-1}\left(J_{1} H_{R}\right)=J_{1} H_{R}^{\prime}$, and $\bar{h}_{i}=\bar{h}_{i}^{\prime}, i=1, \cdots, n-1$, with $\bar{h}_{n}=2 \bar{h}_{n}^{\prime}$. If $J_{1}$ contains 2 , then writing $h=\sum n_{i} \bar{h}_{i}$ in $C^{-1}\left(J_{1} H_{R}\right)$, we have $n_{n} \in(1 / 2) J_{1}=R$. The same is true if $J_{1}$ fails to contain 2 , except that $(1 / 2) J_{1}=J_{1}$. In the latter case, $M_{1}=J_{1} L_{R}$; in the former $M_{1}=J_{1} E_{R}+J_{1} \bar{h}_{1}+\cdots+J_{1} \bar{h}_{n-1}+R \bar{h}_{n}$. Now for any $R$-module $N^{\prime}=J^{\prime} E_{L}+\widetilde{H}$ where $2 J_{1} \subseteq J^{\prime} \subseteq J_{1}$ and $J^{\prime} H_{L}+J_{1} H_{S} \subseteq \widetilde{H} \subseteq C^{-1}\left(J^{\prime} H_{L}+J_{1} H_{S}\right), N^{\prime}+J_{1} E_{S}$ will be an ideal in $L_{R}$. The first step in constructing $M_{2}$ then is to find a $J_{2}$ in $R$ maximal among the $R$-ideals contained in $J_{1}$ which also contain $2 J_{1}$. Then one constructs $M_{2}$ and the next few $M_{i}$ by determining which $\widetilde{H}$ can be fitted into a composition series through $C^{-1}\left(J_{2} H_{L}+J_{1} H_{S}\right)$ so as to contain $J_{2} H_{L}+J_{1} H_{S}$. Then the whole process breaks into two possibilities. One either constructs an $M$ with $M \cap E_{S}=J_{2} E_{s}$, and repeats the above steps with $J_{2}$ in place of $J_{1}$, or else finds a $J_{3}$ maximal in $J_{2}$ which contains $2 J_{1}$ and looks for additional $\widetilde{H}$. As can be seen, numerous alternative paths exist for finishing the composition series in $L_{R}$ through construction of one in $R$.

\section{REFERENCES}

1. C. Chevalley, Sur certains groupes simples, Tôhoku Math. J. (2) 7 (1955), 14-66.

2. J. Dieudonné, Les algèbres de Lie simples associées aux groupes simples algébriques sur un corps de charactéristique $p>0$, Rend. Circ. Mat. Palermo (2) 6 (1957), 198-204. 3. J. Hurley, Ideals in Chevalley algebras, Trans. Amer. Math. Soc. 137 (1969), 245258.

4. — Extensions of Chevalley algebras (to appear)

5. N. Jacobson, Classes of restricted Lie algebras of characteristic $p$ I, Amer. J. Math. 63 (1941), 481-515.

6. - Restricted Lie algebras of characteristic p, Trans. Amer. Math. Soc. 50 (1941), 15-25.

7. T. Ono, Sur les groupes de Chevalley, J. Math. Soc. Japan 10 (1958), 307-313.

8. R. Steinberg, Automorphisms of classical Lie algebras, Pacific J. Math. 11 (1961), 1119-1129.

9. O. Zariski and P. Samuel, Commutative algebra, Volume 1, Princeton, Van Nostrand, 1958.

Received May 16, 1969.

UNiversity of CALIFoRNia, Riverside 


\section{PACIFIC JOURNAL OF MATHEMATICS}

\section{EDITORS}

\author{
H. SAMELSON \\ Stanford University \\ Stanford, California 94305 \\ RICHARD PIERCE \\ University of Washington \\ Seattle, Washington 98105
}

J. DugundJI

Department of Mathematics University of Southern California Los Angeles, California 90007

BASIL GORDON*

University of California

Los Angeles, California 90024

\section{ASSOCIATE EDITORS}
E. F. BECKENBACH
B. H. NEUMANN
F. WOLE
K. YOSHIDA

\section{SUPPORTING INSTITUTIONS}

\author{
UNIVERSITY OF BRITISH COLUMBIA \\ CALIFORNIA INSTITUTE OF TECHNOLOGY \\ UNIVERSITY OF CALIFORNIA \\ MONTANA STATE UNIVERSITY \\ UNIVERSITY OF NEVADA \\ NEW MEXICO STATE UNIVERSITY \\ OREGON STATE UNIVERSITY \\ UNIVERSITY OF OREGON \\ OSAKA UNIVERSITY \\ UNIVERSITY OF SOUTHERN CALIFORNIA
}

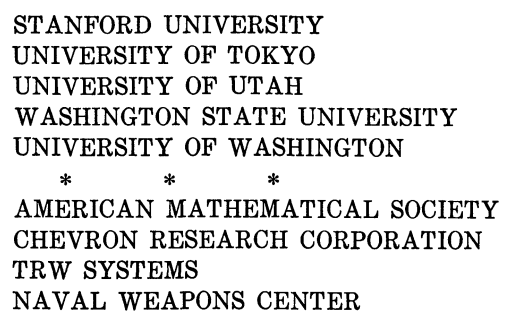

The Supporting Institutions listed above contribute to the cost of publication of this Journal, but they are not owners or publishers and have no responsibility for its content or policies.

Mathematical papers intended for publication in the Pacific Journal of Mathematics should be in typed form or offset-reproduced, (not dittoed), double spaced with large margins. Underline Greek letters in red, German in green, and script in blue. The first paragraph or two must be capable of being used separately as a synopsis of the entire paper. The editorial "we" must not be used in the synopsis, and items of the bibliography should not be cited there unless absolutely necessary, in which case they must be identified by author and Journal, rather than by item number. Manuscripts, in duplicate if possible, may be sent to any one of the four editors. Please classify according to the scheme of Math. Rev. 36, 1539-1546. All other communications to the editors should be addressed to the managing editor, Richard Arens, University of California, Los Angeles, California, 90024.

50 reprints are provided free for each article; additional copies may be obtained at cost in multiples of 50 .

The Pacific Journal of Mathematics is published monthly. Effective with Volume 16 the price per volume (3 numbers) is $\$ 8.00$; single issues, $\$ 3.00$. Special price for current issues to individual faculty members of supporting institutions and to individual members of the American Mathematical Society: $\$ 4.00$ per volume; single issues $\$ 1.50$. Back numbers are available.

Subscriptions, orders for back numbers, and changes of address should be sent to Pacific Journal of Mathematics, 103 Highland Boulevard, Berkeley, California, 94708.

PUBLISHED BY PACIFIC JOURNAL OF MATHEMATICS, A NON-PROFIT CORPORATION

Printed at Kokusai Bunken Insatsusha (International Academic Printing Co., Ltd.), 7-17, Fujimi 2-chome, Chiyoda-ku, Tokyo, Japan.

* Acting Managing Editor. 


\section{Pacific Journal of Mathematics}

\section{Vol. 32, No. $2 \quad$ February, 1970}

Harry P. Allen and Joseph Cooley Ferrar, Jordan algebras and exceptional subalgebras of the exceptional algebra $E_{6} \ldots \ldots \ldots \ldots \ldots \ldots \ldots 283$

David Wilmot Barnette and Branko Grünbaum, Preassigning the shape of a

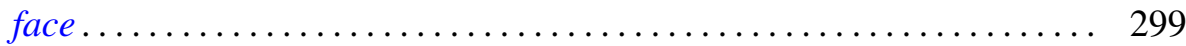

Robert Francis Craggs, Involutions of the 3-sphere which fix 2-spheres . . . . 307

David William Dean, Bor-Luh Lin and Ivan Singer, On k-shrinking and $k$-boundedly complete bases in Banach spaces ................ 323

Martin Engert, Finite dimensional translation invariant subspaces ....... 333

Kenneth Lewis Fields, On the global dimension of residue rings ......... 345

Howard Gorman, The Brandt condition and invertibility of modules ....... 351

Benjamin Rigler Halpern, A characterization of the circle and interval ..... 373

Albert Emerson Hurd, A uniqueness theorem for second order quasilinear

hyperbolic equations ............................... 415

James Frederick Hurley, Composition series in Chevalley algebras ...... 429

Meira Lavie, Disconjugacy of linear differential equations in the complex

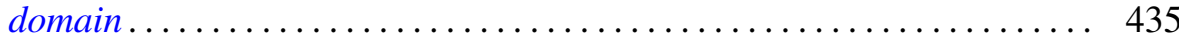

Jimmie Don Lawson, Lattices with no interval homomorphisms ......... 459

Roger McCann, A classification of center-foci ................. 467

Evelyn Rupard McMillan, On continuity conditions for functions . . . . . . . 479

Graciano de Oliveira, A conjecture and some problems on permanents .... 495

David L. Parrott and S. K. Wong, On the Higman-Sims simple group of order $44,352,000$.

Jerome L. Paul, Extending homeomorphisms ................. 517

Thomas Benny Rushing, Unknotting unions of cells .............. 521

Peter Russell, Forms of the affine line and its additive group.......... 527

Niel Shilkret, Non-Archimedean Gelfand theory ................. 541

Alfred Esperanza Tong, Diagonal submatrices of matrix maps.......... 551 